\title{
Lasers in Medical Science
}

\section{Photodynamic Inactivation of Klebsiella pneumoniae Biofilms and Planktonic cells by 5- Aminolevulinic Acid and 5-Aminolevulinic Acid Methyl Ester \\ --Manuscript Draft--}

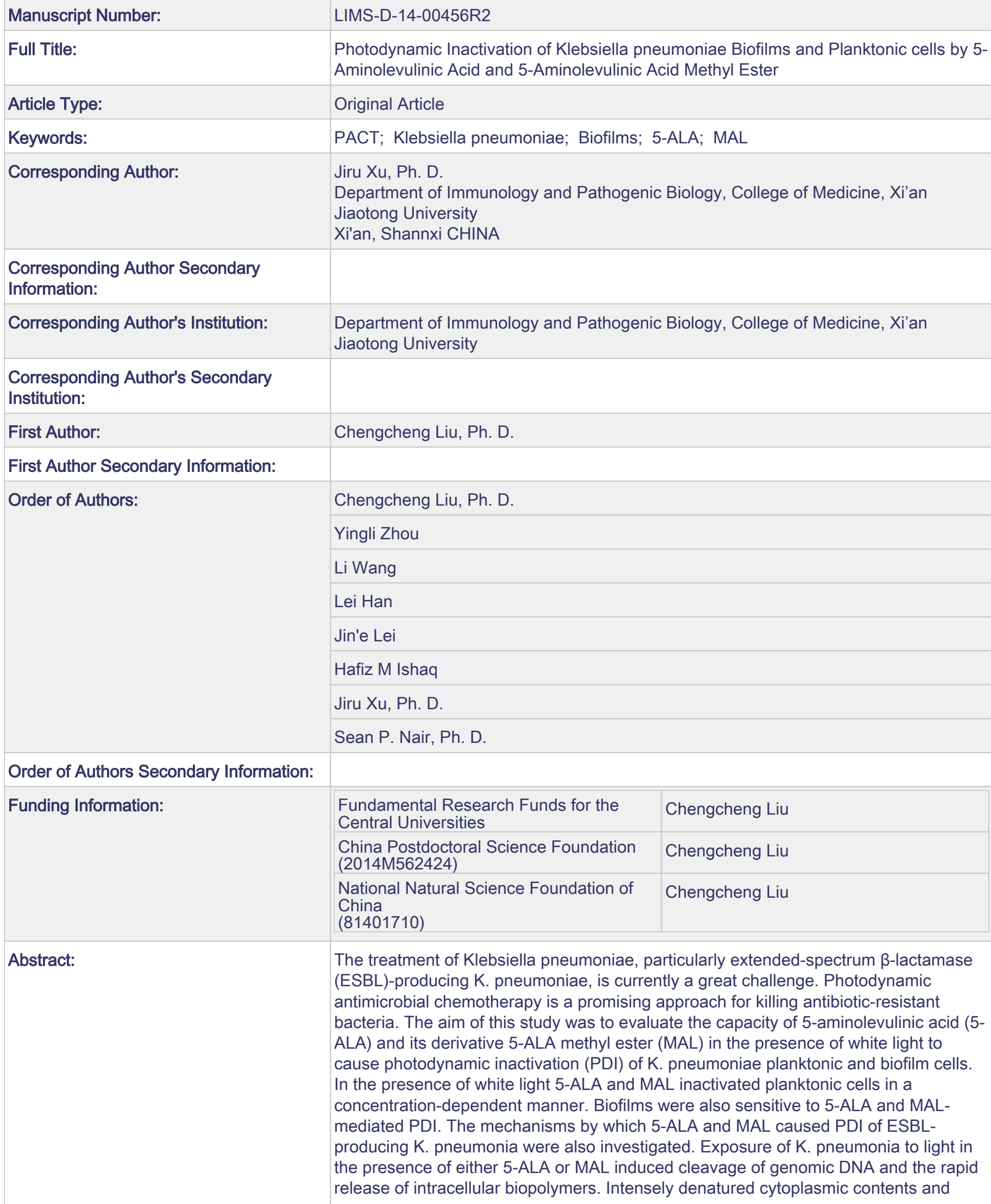


aggregated ribosomes were also detected by transmission electron microscopy.

Scanning electron microscopy showed that PDI of biofilms caused aggregated bacteria to detach and that the bacterial cell envelope was damaged. This study provides insights into 5-ALA and MAL-mediated PDI of ESBL-producing K. pneumoniae. 


\title{
Photodynamic Inactivation of Klebsiella pneumoniae Biofilms and Planktonic cells by 5-Aminolevulinic Acid and
}

\section{5-Aminolevulinic Acid Methyl Ester}

\begin{abstract}
The treatment of Klebsiella pneumoniae, particularly extended-spectrum $\beta$-lactamase (ESBL)-producing $K$. pneumoniae, is currently a great challenge. Photodynamic antimicrobial chemotherapy is a promising approach for killing antibiotic-resistant bacteria. The aim of this study was to evaluate the capacity of 5-aminolevulinic acid (5-ALA) and its derivative 5-ALA methyl ester (MAL) in the presence of white light to cause photodynamic inactivation (PDI) of K. pneumoniae planktonic and biofilm cells. In the presence of white light 5-ALA and MAL inactivated planktonic cells in a concentration-dependent manner. Biofilms were also sensitive to 5-ALA and MAL-mediated PDI. The mechanisms by which 5-ALA and MAL caused PDI of ESBL-producing $K$. pneumonia were also investigated. Exposure of $K$. pneumonia to light in the presence of either 5-ALA or MAL induced cleavage of genomic DNA and the rapid release of intracellular biopolymers. Intensely denatured cytoplasmic contents and aggregated ribosomes were also detected by transmission electron microscopy. Scanning electron microscopy showed that PDI of biofilms caused aggregated bacteria to detach and that the bacterial cell envelope was damaged. This study provides insights into 5-ALA and MAL-mediated PDI of ESBL-producing K. pneumoniae.
\end{abstract}

Keywords PACT, Klebsiella pneumoniae, Biofilms, 5-ALA, MAL

\section{Introduction}

Klebsiella pneumoniae can cause serious infections such as liver or splenic abscess, pneumonia, empyema, and endophthalmitis [1]. Due to the extensive use of antibiotics, extended-spectrum $\beta$-lactamase (ESBL)-producing $K$. pneumoniae is now 
a serious public health concern [2]. Since ESBLs can hydrolyse many $\beta$-lactam antibiotics, such as penicillins and expanded-spectrum cephalosporins, carbapenems which are resistant to hydrolysis, are often used to treat patients who have in cases serious infections with ESBL-producing $K$. pneumonia [3]. However, the increasing rate of carbapenem resistance in recent years necessitates the development of alternative approaches for treating ESBL-producing $K$. pneumoniae infections [4].

One promising approach against antibiotic-resistant bacteria is photodynamic antimicrobial chemotherapy (PACT). This involves the use of a photosensitizer (PS) which upon illumination with light of an appropriate wavelength is excited from a ground state to a triplet state. The triplet state PS reacts with oxygen present in and around the bacteria, resulting in the formation of singlet oxygen or other reactive-oxygen species (ROS) [5]. The ROS can destroy the plasma membrane and other biopolymers, thus resulting in non-specific killing of bacteria [6, 7]. The non-specific nature of the inactivation of bacteria by singlet oxygen and ROS makes it unlikely that bacteria will develop resistance and thus photodynamic therapy is seen as a promising alternative to current antimicrobial agents [8]. Previous studies have reported that $K$. pneumoniae is sensitive to lethal photodynamic inactivation (PDI) mediated by a variety of PSs, such as toluidine blue O [9], methylene blue [10], and rose bengal [11].

In addition to PSs, other molecules such as 5-aminolevulinic acid (5-ALA), which are not in their own right PSs, but are precursors for PSs, are gaining interest. 5-ALA is a metabolic intermediate produced by a rate limiting step in the tetrapyrrole biosynthetic pathway which is essential for production of porphyrins such as haem. When provided exogenously to cells 5-ALA uptake results in the over production and accumulation of photoactive porphyrins since the pathway is no longer rate limited. Uptake of 5-ALA and subsequent synthesis and accumulation of porphyrins is highest in cells that are rapidly dividing, with high metabolic activity, which results in selectivity towards bacteria over normal host cells of the cell [12]. Recently, 5-ALA-mediated PACT has been investigated to inactivate several important antibiotic-resistant bacteria, including methicillin-resistant Staphylococcus aureus 
(MRSA) [13, 14] and multidrug-resistant (MDR) Escherichia coli [14, 15]. A previous study reported by Yow et al. demonstrated that 5-ALA was not effective at mediating PDI of $K$. pneumoniae [16]. However the methodology used by these authors was not optimal because 5-ALA is not a PS, it is a precursor used by cells to generate endogenous PS. Therefore in this study we have reexamined the potential of 5-ALA and its derivative 5-ALA methyl ester (MAL) to mediate the PDI of three $K$. pneumoniae strains, either grown as biofilms or as planktonic cultures. The mechanisms by which PDI of ESBL-producing $K$. pneumonia occurred were also investigated.

\section{Materials and methods}

Bacterial strains and culture conditions

Three K. pneumoniae strains were obtained from the First Affiliated Hospital of Xi'an Jiaotong University, Xi'an, China: ATCC 700603, a clinical isolate of non-ESBL-producing $K$. pneumoniae, and a clinical isolate of ESBL-producing $K$. pneumoniae. Tryptone Soy Agar (TSA) and Tryptone Soy Broth (TSB) were obtained from Shanghai Biotech, China, and Qingdao Rishui Biotech, China, respectively. For the experiments, a single colony from TSA plates was inoculated into $10 \mathrm{~mL}$ TSB and incubated at $37^{\circ} \mathrm{C}$ for $24 \mathrm{~h}$. A $100-\mu \mathrm{L}$ aliquot of the culture was transferred into $10 \mathrm{~mL}$ of fresh TSB and incubated at $37^{\circ} \mathrm{C}$ to attain log-phase growth. The pellets were harvested by centrifugation (4000 rpm for $10 \mathrm{~min}$, Thermo Fisher D-37520, Germany), followed by washing three times with phosphate-buffered saline (PBS, pH 7.4). The pellets were resuspended and diluted with PBS or TSB to a density of $1 \times$ $10^{7}$ colony forming units $(\mathrm{CFU}) / \mathrm{mL}$ prior to the experiments.

Chemicals and light source

5-ALA and MAL were purchased from Sigma-Aldrich, China, and TCI, China, respectively. Stock solutions $(10 \mathrm{mM})$ were freshly prepared by dissolving 5-ALA or MAL in PBS or TSB and were stored in the dark. The solutions were filtered through a $0.22-\mu \mathrm{m}$ filter disk before use. All illuminations were performed with white light 
from a $150 \mathrm{~W}$ xenon lamp (Ceaulight CEL-HXF300, China). A wavelength range between 400 and $780 \mathrm{~nm}$ was selected by optical filters. To avoid heating the samples, the light was passed through a $1-\mathrm{cm}$ water filter. The fluence rate at the level of the samples was $100 \mathrm{~mW} \mathrm{~cm}^{-2}$, as measured with a power meter (Ceaulight CEL-NP2000, China).

PDI on bacteria

Samples of the bacterial suspension $\left(1 \mathrm{~mL}, 1 \times 10^{7} \mathrm{CFU} / \mathrm{mL}\right)$ were centrifuged at $4000 \mathrm{rpm}$ for $10 \mathrm{~min}$. The pellets were resuspended in $1 \mathrm{~mL}$ of ALA or MAL solutions of varying concentrations and incubated in the dark at $37^{\circ} \mathrm{C}$ for $4 \mathrm{~h}$ in a shaking incubator $(100 \mathrm{rpm})$. The samples were transferred to sterile $35-\mathrm{mm}$ polystyrene culture dishes and irradiated for $60 \mathrm{~min}$. After irradiation, bacterial suspensions were centrifuged at $4000 \mathrm{rpm}$ for $10 \mathrm{~min}$. The pellets were resuspended and serially diluted 10 -fold with s PBS, and $20 \mu \mathrm{L}$ of each dilution was spread in triplicate on TSA. Colonies were counted after $24 \mathrm{~h}$ incubation at $37^{\circ} \mathrm{C}$. The fraction of surviving bacteria was calculated as the $\mathrm{CFU} / \mathrm{mL}$ after exposure to light divided by the $\mathrm{CFU} / \mathrm{mL}$ prior to light exposure.

\section{Biofilm formation}

The tissue culture plate method [17] was used to screen for biofilm formation. Bacterial suspensions were diluted to $1 \times 10^{6} \mathrm{CFU} / \mathrm{mL}$ in TSB and $200 \mu \mathrm{L}$ of the diluted suspension was inoculated into the wells of a 96-well polystyrene microplate (Corning, USA). After incubation at $37^{\circ} \mathrm{C}$ for $24 \mathrm{~h}$ the medium was removed and the biofilms were washed twice with PBS. The biofilm was fixed with 95\% ethanol for 10 min and stained with $200 \mu \mathrm{L}$ of a $0.1 \%(\mathrm{w} / \mathrm{v})$ aqueous solution of crystal violet for 15 min at room temperature. The wells were washed twice with PBS to remove excess stain. After drying at $37^{\circ} \mathrm{C}$ for $2 \mathrm{~h}$, biofilm formation was quantified by solubilisation of the crystal violet stain in $200 \mu \mathrm{L}$ of $30 \%$ (w/v) glacial acetic acid for 10 min with shaking at $200 \mathrm{rpm}$, and absorbance was read on a multidetection microplate reader (Thermo Fisher 1510, Finland) at $492 \mathrm{~nm}\left(\mathrm{~A}_{492 \mathrm{~nm}}\right)$. $\mathrm{A}_{492 \mathrm{~nm}}>0.240$ was indicative of 
biofilm formation.

PDI of the bacterial biofilm

A bacterial suspension $\left(2 \mathrm{~mL} ; 1 \times 10^{6} \mathrm{CFU} / \mathrm{mL}\right)$ was inoculated into the wells of a sterile 24-well polystyrene microplate (Corning, USA) that contained sterile glass coverslips and incubated at $37^{\circ} \mathrm{C}$ for $24 \mathrm{~h}$. The culture medium was removed and the biofilms on coverslips were gently washed twice with PBS to remove loosely adherent bacteria. Then, the coverslips were removed with sterilized forceps and placed into the wells of a new 24-well microplate containing $2 \mathrm{~mL} 10 \mathrm{mM} \mathrm{5-ALA} \mathrm{or}$ MAL. The microplate was incubated at $37^{\circ} \mathrm{C}$ for $4 \mathrm{~h}$ in the dark. After irradiation for $60 \mathrm{~min}$, the coverslips were gently washed twice with PBS and placed into the wells of another 24-well microplate. The biofilms were resuspended in $2 \mathrm{~mL}$ of PBS and dislodged by ultrasonication (Hangzhou Front Ultrasoni FRQ-1002T, China) for 10 min, followed by rapid vortexing with a vortex mixer (Haimen Qilinbeier QL-901, China) for 1 min. Bacterial suspensions were serially 10-fold diluted with PBS, and $20 \mu \mathrm{L}$ of each dilution was plated in triplicate on TSA. Colonies were counted after 24 $\mathrm{h}$ incubation at $37^{\circ} \mathrm{C}$.

Genomic DNA purification and electrophoresis

To determine if DNA damage was one of the mechanisms behind PDI of ESBL-producing $K$. pneumonia, genomic DNA was extracted and analysed by agarose gel electrophoresis. After irradiation, genomic DNA was immediately extracted from the bacteria using a Genomic DNA Purification Kit (Promega, USA). DNA samples were gently mixed with $6 \mathrm{X}$ loading-buffer $(0.25 \% \mathrm{w} / \mathrm{v}$ bromophenol blue, $40 \% \mathrm{w} / \mathrm{v}$ sucrose, $1.15 \%$ acetic acid, $40 \mathrm{mM}$ Tris, $1 \mathrm{mM}$ EDTA), and were analysed by electrophoresis in a 1\% agarose gel in Tris/Borate/EDTA buffer (TBE, 90 $\mathrm{mM}$ Tris- $\mathrm{HCl}, 90 \mathrm{mM}$ boric acid, and $2 \mathrm{mM}$ EDTA, $\mathrm{pH}$ 8) at $2.9 \mathrm{~V} \mathrm{~cm}^{-1}$ for $1.5 \mathrm{~h}$. Ethidium bromide $(1 \mu \mathrm{g} / \mathrm{mL})$ was incorporated into the agarose gel. A Lambda DNA/HindIII digest marker (Promega, USA) was used as the molecular weight marker, with DNA fragments between 125 to 23,130 bp. 
Integrity of cell membrane

The integrity of the cell membrane was examined by measuring the release of materials absorbing at $260 \mathrm{~nm}$ [18-21]. After irradiation, ESBL-producing $K$. pneumoniae was centrifuged at $4000 \mathrm{rpm}$ for $10 \mathrm{~min}$. The pellets were discarded and the material released into the supernatant was measured by scanning UV-Visible spectroscopy (Agilent 8453, USA) at room temperature.

Transmission electron microscopy (TEM)

TEM samples were prepared according to a previously described method [21, 22]. After irradiation, ESBL-producing K. pneumoniae was centrifuged at $4000 \mathrm{rpm}$ for 10 min and fixed in $2.5 \%$ glutaraldehyde (SCRC, China) at $4^{\circ} \mathrm{C}$ for $2 \mathrm{~h}$. The pellets were washed with PBS three times and fixed in 1\% osmium tetraoxide (Johnson Matthey, England) at $4{ }^{\circ} \mathrm{C}$ for $2 \mathrm{~h}$. The pellets were dehydrated with gradients of ethanol and embedded in Epon 812 epoxy resin (SPI-Chem, USA) at $60^{\circ} \mathrm{C}$ for $24 \mathrm{~h}$. Thin-section samples of 50-70 nm were prepared using a LKB-V ultratome (LKB, Sweden). The samples were stained with uranyl acetate and lead citrate for $15 \mathrm{~min}$. Finally, the samples were viewed and digitally photographed using a TEM (Hitachi H-7650, Japan).

Scanning electron microscopy (SEM) analysis of biofilms

SEM was used to observe morphologic alterations in bacterial biofilms. Biofilms of ESBL-producing $K$. pneumoniae on coverslips were washed with PBS three times and fixed in $2.5 \%$ glutaraldehyde at $4^{\circ} \mathrm{C}$ for $2 \mathrm{~h}$. Then, the coverslips were washed with PBS three times and fixed in $1 \%$ osmium tetraoxide at $4^{\circ} \mathrm{C}$ for $2 \mathrm{~h}$. After dehydration with gradients of ethanol, the samples were freeze-dried, sputter-coated with gold, and observed using a SEM (Hitachi TM-1000, Japan).

\section{Results}

PDI of planktonic cells

5-ALA did not exhibit obvious dark toxicity for the three $K$. pneumoniae strains at the 
concentrations tested (Fig. 1). In contrast, the irradiated groups showed reduced bacterial survival with increasing concentrations of 5-ALA. When a concentration of $10 \mathrm{mM}$ 5-ALA was used, 3.68, 3.17, and $3.20 \log _{10}$ reductions in the surviving fraction were observed for ATCC 700603, the non-ESBL-producing clinical isolate, and the ESBL-producing clinical isolate, respectively. MAL induced some dark toxicity in a concentration dependent manner. In the dark 10 mM MAL caused 1.04, 0.92, and $0.96 \log _{10}$ reductions in the surviving fraction of ATCC 700603, the non-ESBL-producing clinical isolate, and the ESBL-producing isolate, respectively. After irradiation of MAL treated cells, there were reductions in bacterial survival of 4.80, 4.32, and $4.52 \log _{10}$ for ATCC 700603, the non-ESBL-producing clinical isolate, and the ESBL-producing isolate, respectively. All of the strains were also irradiated without 5-ALA or MAL treatment and this did not cause any change in bacterial survival.

PDI of biofilms

In the 96 well tissue culture plate analysis of biofilm formation $\mathrm{A}_{492 \mathrm{~nm}}$ values of 0.73 , 2.07, and 1.39 were obtained for ATCC 700603, the non-ESBL-producing clinical isolate, and the ESBL-producing clinical isolate, respectively. These values exceeded

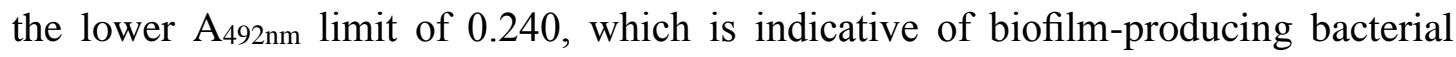
strains. As shown in Fig. 2, no significant difference in the surviving fraction was found for the biofilms treated with 5-ALA or MAL in the dark compared to untreated biofilms $(P>0.05)$. After treatment with $10 \mathrm{mM}$ 5-ALA and irradiation for $60 \mathrm{~min}$, $3.09,1.92$, and $2.28 \log _{10}$ reductions in the surviving fraction were achieved for the biofilms of ATCC 700603, the non-ESBL-producing clinical isolate, and the ESBL-producing clinical isolate, respectively. Greater reductions in the surviving fraction of bacteria in biofilms were seen after treatment with $10 \mathrm{mM}$ MAL and irradiation for $60 \mathrm{~min}$, with 4.25, 3.49, and $3.91 \log _{10}$ reductions observed for ATCC 700603, the non-ESBL-producing clinical isolate, and the ESBL-producing clinical isolate, respectively. These results demonstrate that biofilms formed by the tested strains are sensitive to the 5-ALA and MAL-mediated PDI. 
Photodynamic effect on genomic DNA

As shown in Fig. 3, the genomic DNA isolated from the ESBL-producing $K$. pneumoniae strain incubated with 5-ALA or MAL in the dark migrated the same distance as DNA from untreated cells suggesting that these molecules do not cause macroscale DNA damage. However after irradiation of 5-ALA or MAL treated cells degradation of genomic DNA was observed indicating that 5-ALA and MAL-mediated PDI induced DNA damage in ESBL-producing K. pneumoniae.

Leakage of intracellular biopolymers

As an indication of membrane damage the release of intracellular components was monitored by scanning spectroscopy. The results of scanning UV-Vis spectroscopy are shown in Fig. 4. The absorbance at $260 \mathrm{~nm}$ of the supernatant from the untreated bacteria was the same as the supernatants from bacteria incubated with 5-ALA or MAL in the dark (the inner plot). A slight increase in the absorption at $260 \mathrm{~nm}$ of the supernatant obtained from bacteria which had been sonicated for $15 \mathrm{~min}$ was observed. The supernatants from bacteria which had been treated with $10 \mathrm{mM}$ 5-ALA or MAL and irradiated for 60 min exhibited a large increase in absorption at $260 \mathrm{~nm}$, suggesting that the functionality of the membrane had been impaired allowing the release of intracellular biopolymers.

Photodynamic effect on cellular structure

The ESBL-producing $K$. pneumoniae clinical isolate was analysed by TEM to determine if the photodynamic effect mediated by 5-ALA and MAL caused any cell structure changes at the morphological level. Representative results are shown in Fig. 5. The normal rod-shaped structure was observed for bacteria which had not been treated (Fig. 5 A) and for cells treated with 5-ALA or MAL in the dark (Fig. 5 B and Fig. 5 C). In these images the cell wall and plasma membrane are well differentiated. The ribosomes are dispersed in the cytoplasm, dark particles, with nucleic acid differentiated as a clear zone. Fig. $5 \mathrm{D}$ and $5 \mathrm{E}$ show images of bacteria treated with $10 \mathrm{mM}$ 5-ALA or MAL and irradiated for $60 \mathrm{~min}$, respectively. Damage to the 
bacterial cells can be seen in these images with condensation of ribosomes resulting in a low-density area in the centre of the bacterial cells and in the case of Fig $5 \mathrm{E}$ filamentous structures in the cell centre.

SEM results

Biofilms of the ESBL-producing $K$. pneumoniae clinical isolate were analysed by SEM to determine if the photodynamic effect mediated by 5-ALA and MAL caused structural changes in the biofilm community. As shown in Fig. 6 A-C, large cellular aggregates surrounded by extracellular matrix were observed for the biofilms without any treatment and for the biofilms incubated with 5-ALA or MAL in the dark. Treatment of biofilms with $10 \mathrm{mM}$ 5-ALA or MAL and irradiation for 60 min caused biofilm disruption with only small clusters of cells or individually adherent cells remaining (Fig. $6 \mathrm{D}$ and $6 \mathrm{E}$ ). In addition some changes in the appearance of these bacteria could be observed. As shown in Fig. 6 D flaws (white arrow) and concavities giving different cell shapes (black arrow) could be observed in individual bacteria. In Fig. 6 E, hollow cracks (white arrows) could be observed on the surface of some bacteria. These results indicated that the cell envelope was significantly damaged during PDI.

\section{Discussion}

The biosynthesis of 5-ALA is a rate limiting step downstream of haem biosynthesis and exists in nearly all cells. Unlike eukaryotes in which ALA is synthesized by the enzyme aminolevulinate sythetase by condensation of glycine and succinyl coenzyme $\mathrm{A}$, in most bacteria ALA is synthesized via the C5-pathway utilizing glutamate as a precursor. The next steps in the biosynthesis of porphyrins involve the condensation of two molecules of 5-ALA to form porphobilinogen, in a reaction catalysed by 5-ALA dehydratase, followed by the condensation of four molecules of porphobilinogen to form a linear tetrapyrrole, which cyclizes to form uroporphyrinogens I and III. Bacteria can use uroporphyrinogen III as an intermediate in the synthesis of various photoactive porphyrins such as uroporphyrin III, 
coproporphyrin III, and protoporphyrin IX. Because the biosynthesis of 5-ALA is the rate limiting step in these biosynthetic pathways, when an excessive amount of this molecule is provided exogenously and taken up by cells, photoactive porphyrins accumulate, particularly in rapidly growing cells such as bacteria thereby giving a degree of selective targeting [12].

One issue with the use of 5-ALA is that it does not transverse cell membranes and other biological barriers efficiently, and to counter this several more lipophilic derivatives have been developed to improve bioavailability [23]. Fotinos et al. investigated the capacity of 5-ALA and 5-ALA esters, with increasing chain lengths, from methyl ester to octyl ester and hence increasing lipophilicities, to cause the accumulation of photoactive porphyrins in bacteria and to mediate PDI. They found that the dark toxicities of the 5-ALA derivatives increased with their lipophilicity [14]. 5-ALA pentyl ester, hexyl ester, and octyl ester did not induce photoactive porphyrin accumulation nor did they mediate effective PDI, the authors attributed this finding to the strong dark toxicity of these molecules. In the case of MAL these authors only observed dark toxicity at higher concentrations, which is in accordance with our findings reported here. Fotinos et al also investigated the capacity of 5-ALA and MAL to mediated PDI of different bacterial species and strains, and found that the PDI varied with the bacterial strain. The gram-positive strains appeared to be significantly more sensitive to 5-ALA-mediated PDI than the gram-negative strains [14]. Yow et al. compared the capacity of methylene blue (MB) and 5-ALA to mediate PDI of $K$. pneumoniae and ESBL-producing $K$. pneumoniae strains [16]. They demonstrated a $5.9 \log _{10}$ reduction in the viability of an ESBL-nonproducing clinical isolate of $K$. pneumoniae and $4.8 \log _{10}$ reduction of an ESBL-producing $K$. pneumoniae with $10 \mu \mathrm{M}$ MB. However they found that ALA was ineffective at mediating PDI of $K$. pneumoniae at the highest concentration tested, $6 \mu \mathrm{M}$. In contrast, here we report that $K$. pneumonia is in fact sensitive to ALA mediated PDI. The difference between the findings of Yow et al. and the results we present here are probably due to the fact that they used very low concentrations of 5-ALA. Another difference between the two studies is that the bacterial cell density we used was lower 
than that used by Yow et al and it has been previously been reported that this can affect the efficiency of PDI, albeit for different bacteria [24]. Our data and that of Fotinos et al., where they treated two E. coli strains (K12 and a uropathogenic isolate) with 0.1 and $1.0 \mathrm{mM} \mathrm{5-ALA} \mathrm{and} \mathrm{upon} \mathrm{irradiation} \mathrm{with} 120 \mathrm{~J} \mathrm{~cm}^{-2}$ of white light achieved 3.31 and $4.30 \log _{10}$ reductions in the survival [14], suggest that $K$. pneumoniae is less susceptible to 5-ALA mediated PDI than E. coli.

Bacterial biofilms are communities of cells, attached to a surface and embedded in extracellular polymeric substances (EPS) [25]. Bacteria in biofilms are more resistant to antibiotics and host immune defenses than their planktonic counter parts, thus hampering treatment and clearance of infections [26]. In addition to causing significant morbidity and mortality biofilm associated infections are also a significant economic burden on healthcare services since they are recalcitrant to antimicrobial therapy and therefore also require the removal of colonized indwelling devices. A previous study has shown that biofilms formed by MRSA are sensitive to 5-ALA-mediated PDI treatment [13]. Lee et al reported that no viable bacteria were detected when Pseudomonas aeruginosa biofilms were treated with $20 \mathrm{mM}$ 5-ALA and exposed to $120 \mathrm{~J} \mathrm{~cm}^{-2}$ of $630 \mathrm{~nm}$ LED light [27]. In the present study PDI of $K$. pneumoniae biofilms mediated by 5-ALA and MAL was also assessed. $K$. pneumoniae strains were susceptible to PDI when grown as biofilms but the magnitude of inactivation was not as great as with planktonic cells. As mentioned microbial biofilms are generally less susceptible than planktonic cells to antimicrobial agents, such as antimicrobial peptides and antibiotics [28], and less susceptible to PDI. Although a number of factors account for increased resistance of biofilm cells to antimicrobial agents, including changes in gene expression, the affinity of a PS for the EPS will also affect PS binding, and ultimately the efficiency of PDI [29]. Our data on 5-ALA mediated PDI of $K$. pneumoniae biofilms where we find that the greatest biofilm formers are the least susceptible to PDI suggests that EPS may have a protective role. The SEM investigation suggested that 5-ALA and MAL-mediated PDI had another important action beyond killing the bacteria in biofilms. After irradiation, the number of adherent bacteria greatly reduced and only a few colonies 
were observed, implying that 5-ALA and MAL-mediated PDI could disrupt the biofilm presumably by destroying EPS which is responsible for bacterial aggregation.

PDI of bacteria has been proposed to occur through inactivation of a number of biomolecules. For example damage to DNA would interfere with chromosome segregation, DNA replication and transcription. PDI of bacteria has been demonstrated to cause breaks in single and double stranded DNA and the disappearance of the supercoiled form of plasmids [30, 31]. However other authors have suggested that although DNA damage occurred, it might not be the prime cause of bacterial death. In support of this Caminos and Choi found that DNA was only slightly degraded after a relatively long period of irradiation, although the bacterial strains were effectively inactivated $[22,32]$. In a study by Nitzan et al. on the PDI of Acinetobacter baumannii and $E$. coli using the cationic PS TMPyP it was found that structural damage to the membrane was induced by the ROS produced upon photosensitization, while DNA breakage appeared only after a long period of irradiation, when the bacterial cells were no longer viable [33]. They concluded that cytoplasmic membrane damage and not DNA breakage was the major cause of bacterial photoinactivation. In the present study, we detected rapid DNA degradation and release of intracellular biopolymers upon PDI treatment, indicating that 60-min irradiation was sufficient to cause damage to the membrane and genomic DNA. Interestingly on a macro-scale examination of bacteria exposed to 5-ALA mediated PDI by TEM, showed damage to the bacterial cells and condensation of cytoplasmic components (Fig. 5 D). However when bacteria exposed to MAL mediated PDI were examined by TEM strikingly different macro-scale changes were observed, with filamentous structures having been formed in low density areas (Fig. 5 E). These results indicate that PDI mediated by 5-ALA and MAL have different effects on cellular structures but this requires further investigation.

\section{Conclusions}

In summary, the present study assessed the efficacy of 5-ALA and MAL to mediate PDI of three $K$. pneumoniae strains in planktonic and biofilm culture. 5-ALA and 
MAL mediated inactivation of the planktonic cultures in a concentration-dependent manner upon irradiation. Biofilms were also sensitive to 5-ALA and MAL-mediated PDI. Furthermore SEM demonstrated that 5-ALA and MAL mediated PDI also caused biofilm disruption. After PDI of ESBL-producing $K$. pneumoniae, obvious cleavage of genomic DNA and a rapid release of intracellular biopolymers were detected. The condensation of cytoplasmic components was also observed by TEM. Although cleavage of genomic DNA was detected, PDI of ESBL-producing $K$. pneumoniae might be predominantly due to cell envelope injury, intracellular biopolymer leakage, and the denaturation of cytoplasmic components.

Acknowledgements We are really grateful for assistance from Professor Kewu Yang at the College of Chemistry and Materials Science, Northwest University, China. This work was supported by the National Natural Science Foundation of China (81401710), China Postdoctoral Science Foundation (2014M562424), and the Fundamental Research Funds for the Central Universities.

Conflict of interest The authors declare no conflict of interest.

\section{References}

1. Shon AS, Bajwa RPS, Russo TA (2013) Hypervirulent Klebsiella pneumoniae: a new and dangerous breed. Virulence 4 (2): 107-118

2. Pitout JDD, Laupland KB (2008) Extended-spectrum $\beta$-lactamase-producing Enterobacteriaceae: an emerging public-health concern Lancet Infect Dis 8 (3): $159-166$

3. Bradford PA (2001) Extended-spectrum $\beta$-lactamases in the 21st century: characterization, epidemiology, and detection of this important resistance threat. Clin Microbiol Rev 14 (4): 933-951

4. Vardakas KZ et al (2012) Carbapenems versus alternative antibiotics for the treatment of bacteraemia due to Enterobacteriaceae producing extended-spectrum $\beta$-lactamases: a systematic review and meta-analysis. J 
Antimicrob Chemother doi: 10. 1093/jac/dks301

5. Denis TGSt et al (2011) All you need is light: Antimicrobial photoinactivation as an evolving and emerging discovery strategy against infectious disease. Virulence 2 (6): 509-520

6. Celli JP et al (2010) Imaging and photodynamic therapy: mechanisms, monitoring, and optimization. Chem Rev 110 (5): 2795-2838

7. Castano AP, Mroz P, Hamblin MR (2006) Photodynamic therapy and anti-tumor immunity. Nat Rev Cancer 6: 535-545

8. Wood S et al (2006) Erythrosine is a potential photosensitizer for the photodynamic therapy of oral plaque biofilms. J Antimicrob Chemother 57 (4): $680-684$

9. Komerik N, Wilson M (2002) Factors influencing the susceptibility of Gram-negative bacteria to toluidine blue O-mediated lethal photosensitization. J Appl Microbiol 92 (4): 618-623

10. Haidaris CG et al (2013) Effective photodynamic therapy against microbial populations in human deep tissue abscess aspirates. Laser Surg Med 45 (8): $509-516$

11. Rossoni RD et al (2010) Comparison of the efficacy of rose bengal and erythrosine in photodynamic therapy against Enterobacteriaceae. Laser Med Sci 25 (4): 581-596

12. Harris F, Pierpoint L (2012) Photodynamic therapy based on 5-aminolevulinic acid and its use as an antimicrobial agent. Med. Res. Rev. 32 (6): 1292-1327

13. Li X et al (2013) Effects of 5-aminolevulinic acid-mediated photodynamic therapy on antibiotic-resistant staphylococcal biofilm: an in vitro study. J Surg Res 184 (2): 1013-1021

14. Fotinos $\mathrm{N}$ et al (2008) Effects on gram-negative and gram-positive bacteria mediated by 5 -aminolevulinic acid and 5-aminolevulinic acid derivatives. Animicrob Agents Chemother 52 (4): 1366-1373

15. Nitzan Y et al (2004) ALA induced photodynamic effects on gram positive and negative bacteria. Photochem Photobiol Sci 3: 430-435 
16. Yow CMN, Fung K, Wong KC (2011) Photodynamic inactivation of multi-drug resistant pathogens in Hong Kong. Hong Kong Med J 17 (Suppl 2): S24-28

17. Peng $\mathrm{Z}$ et al (2011) Quaternized chitosan inhibits icaA transcription and biofilm formation by Staphylococcus on a titanium surface. Antimicrob Agents Chemother 55 (2): 860-866

18. Chen CZ, Cooper SL (2002) Interactions between dendrimer biocides and bacterial membranes. Biomaterials 23 (16): 3359-3368

19. Je JY, Kim SK (2006) Antimicrobial action of novel chitin derivative. Biochim Biophys Acta 1760 (1): 104-109

20. Spesia MB et al (2009) Mechanistic insight of the photodynamic inactivation of Escherichia coli by a tetracationic zinc(II) phthalocyanine derivative. Photodiagn Photodyn Ther 6 (1): 52-61

21. Spesia MB, Duraniti EN (2013) Photodynamic inactivation mechanism of Streptococcus mitis sensitized by zinc (II) 2, 9, 16, 23-tetrakis [2-( $N, N$, $N$-trimethylamino) ethoxy] phthalocyanine. J Photochem Photobiol B 125 (5): $179-187$

22. Caminos DA et al (2008) Mechanisms of Escherichia coli photodynamic inactivation by an amphiphilic tricationic porphyrin and 5, 10, 15, 20-tetra (4- $N$, $N, N$-trimethylammoniumphenyl) porphyrin. Photochem Photobiol Sci 7: $1071-1078$

23. Fotinos $\mathrm{N}$ et al (2006) 5-Aminolevulinic acid derivatives in photomedicine: characteristics, application and perspectives. Photochem Photobiol 82 (4): $994-1015$

24. Demidova T, Hamblin M (2005) Effects of cell-photosensitizer binding and cell density on microbial photoinactivation. Antimicrob Agents Chemother 49 (6): $2329-2335$

25. Sutherland IW (2001) The biofilm matrix-an immobilized but dynamic microbial environment. Trends Microbiol 9 (5): 222-227

26. Stewart PS, Costerton JW (2001) Antibiotic resistance of bacteria in biofilms. Lancet 358 (9276): 135-138 
27. Lee $\mathrm{C}$ et al (2004) 5-Aminolaevulinic acid mediated photodynamic antimicrobial chemotherapy on Pseudomonas aeruginosa planktonic and biofilm cultures. J Photochem Photobiol B 75 (1-2): 21-25

28. Mah TFC, Toole GAO' (2001) Mechanisms of biofilm resistance to antimicrobial agents. Trends Microbiol 9 (1): 34-39

29. Gad F et al (2004) Effects of growth phase and extracellular slime on photodynamic inactivation of gram-positive pathogenic bacteria. Antimicrob Agents Chemother 48 (6): 2173-2178

30. Bertoloni $G$ et al (2000) Photosensitizing activity of hematoporphyrin on Staphylococcus aureus cells. Biochim Biophys Acta 1475 (2): 169-174

31. Capella M, Coelho AM, Menezes S (1996) Effect of glucose on photodynamic action of methylene blue in Escherichia coli cells. Photochem Photobiol 64 (1): $205-210$

32. Choi SS, Lee HK, Chae HS (2012) Comparison of in vitro photodynamic antimicrobial activity of protoporphyrin IX between endoscopic white light and newly developed narrowband endoscopic light against Helicobacter pylori 26695. J Photochem Photobiol B 117 (5): 55-60

33. Nitzan Y, Ashkenazi H (2001) Photoinactivation of Acinetobacter baumannii and Escherichia coli B by cationic hydrophilic porphyrin at various light wavelengths. Curr Microbiol 42 (6): 408-414 


\section{K. pneumoniae (ATCC 700603)}
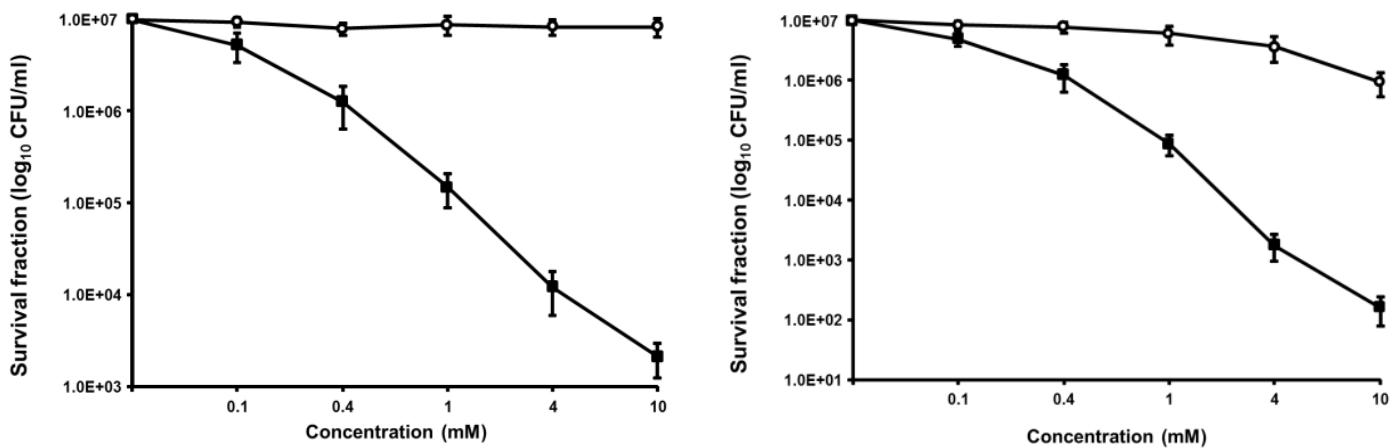

Clinical isolate of non-ESBL-producing $K$. pneumoniae
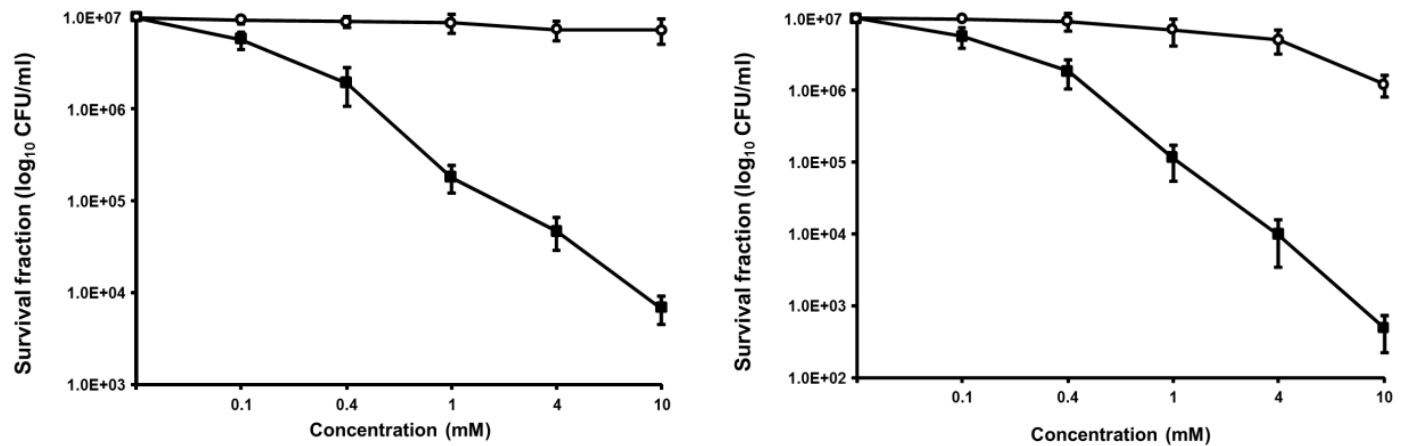

Clinical isolate of ESBL-producing $K$. pneumoniae
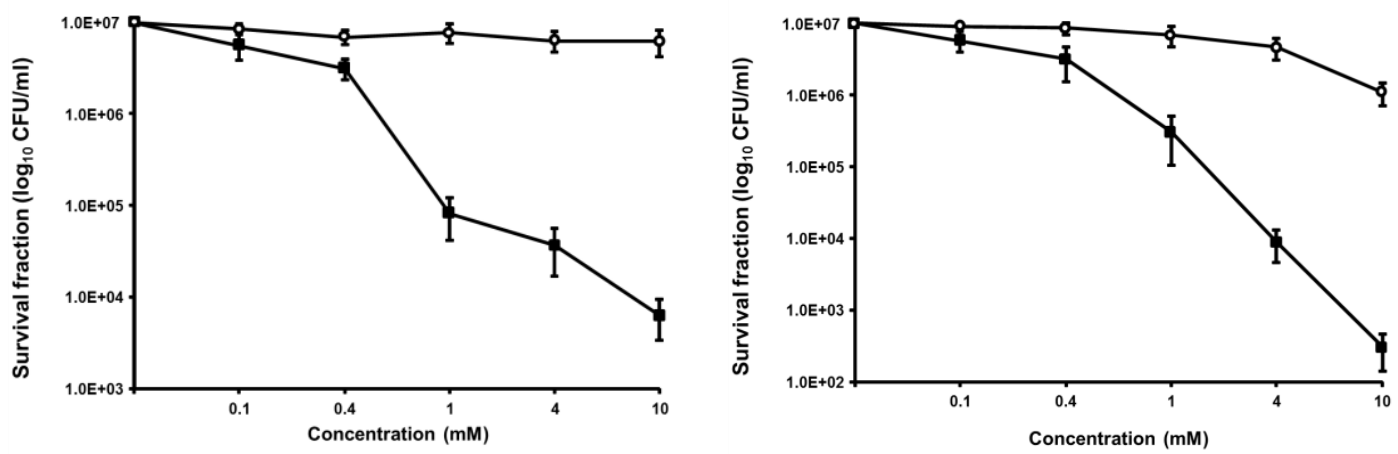

Fig. 1 Bacterial survival curves of $K$. pneumoniae incubated with 5-ALA or MAL at $37^{\circ} \mathrm{C}$ for $4 \mathrm{~h}$ in the dark. Open circles (O) represent the surviving fraction after incubation (dark toxicity), and filled squares ( $\mathbf{m})$ represent the surviving fraction after irradiation by $400-780 \mathrm{~nm}$ white light for $60 \mathrm{~min}$. 


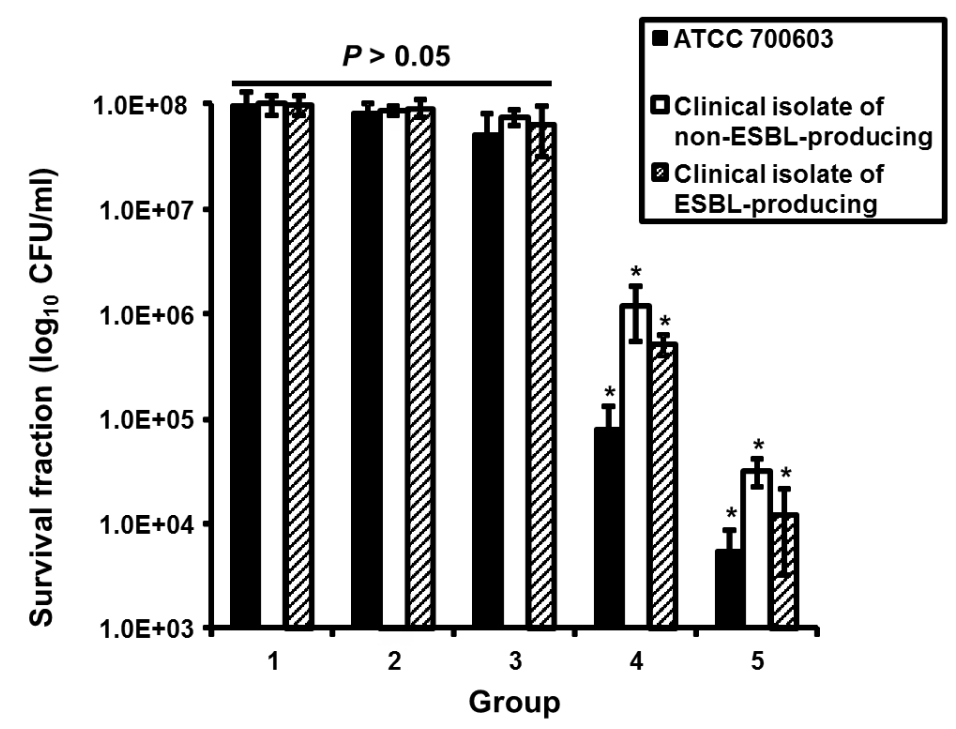

Fig. 2 Surviving fraction of $K$. pneumoniae biofilms after 5-ALA and MAL-mediated PDI treatment. Group 1: without any treatment, group 2: incubated with $10 \mathrm{mM}$ 5-ALA at $37^{\circ} \mathrm{C}$ for $4 \mathrm{~h}$ in the dark, group 3: incubated with $10 \mathrm{mM}$ MAL at $37^{\circ} \mathrm{C}$ for $4 \mathrm{~h}$ in the dark, group 4: incubated with $10 \mathrm{mM}$ 5-ALA and irradiated with 400-780 $\mathrm{nm}$ white light for $60 \mathrm{~min}$, group 5: incubated with $10 \mathrm{mM}$ MAL and irradiated for 60 min. $* P<0.05$ compared with groups $1-3$. 


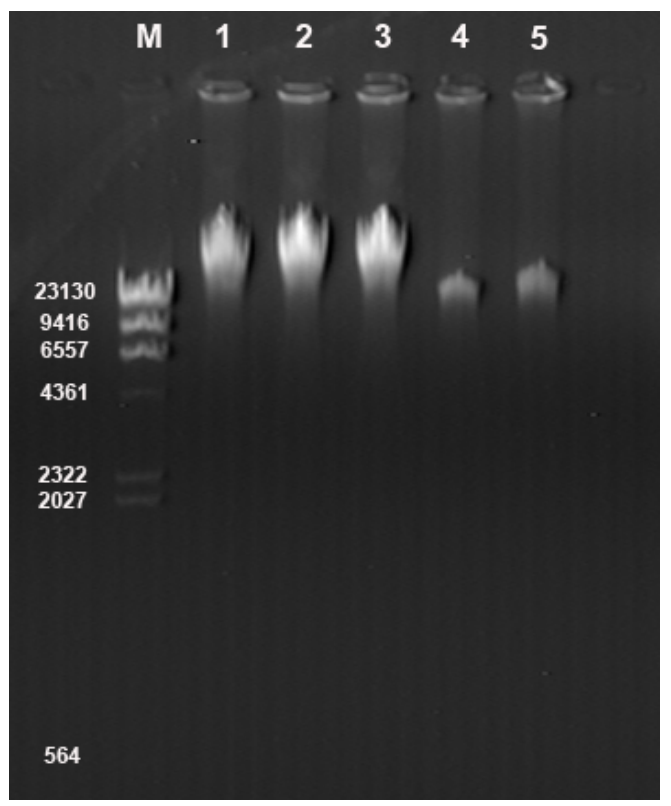

Fig. 3 Agarose gel electrophoresis of genomic DNA samples extracted from ESBL-producing $K$. pneumoniae. Lane M: DNA weight maker, lane 1: without any treatment, lane 2: incubated with $10 \mathrm{mM} \mathrm{5-ALA}$ at $37^{\circ} \mathrm{C}$ for $4 \mathrm{~h}$ in the dark, lane 3: incubated with $10 \mathrm{mM}$ MAL at $37^{\circ} \mathrm{C}$ for $4 \mathrm{~h}$ in the dark, lane 4: incubated with 10 mM 5-ALA and irradiated with 400-780 nm white light for $60 \mathrm{~min}$, lane 5: incubated with $10 \mathrm{mM}$ MAL and irradiated with 400-780 nm white light for $60 \mathrm{~min}$. 


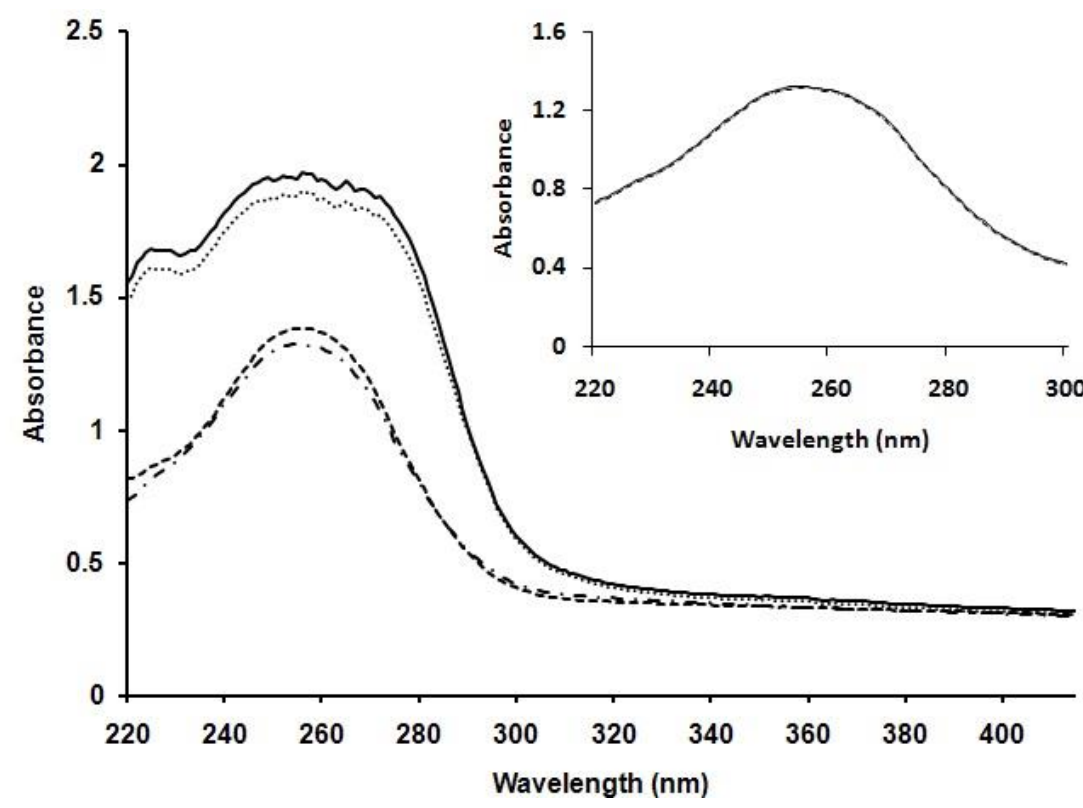

Fig. 4 Release of UV-absorbing material from ESBL-producing $K$. pneumoniae. Dot-dashed line: without any treatment, dashed line: sonicated for $15 \mathrm{~min}$, dotted line: incubated with $10 \mathrm{mM}$ 5-ALA and irradiated with 400-780 nm white light for $60 \mathrm{~min}$, solid line: incubated with $10 \mathrm{mM}$ MAL and irradiated with $400-780 \mathrm{~nm}$ white light for 60 min. Inner plot: solid line: without any treatment, dotted line: incubated with 10 $\mathrm{mM}$ 5-ALA at $37^{\circ} \mathrm{C}$ for $4 \mathrm{~h}$ in the dark, dashed line: incubated with $10 \mathrm{mM}$ MAL at $37^{\circ} \mathrm{C}$ for $4 \mathrm{~h}$ in the dark. 

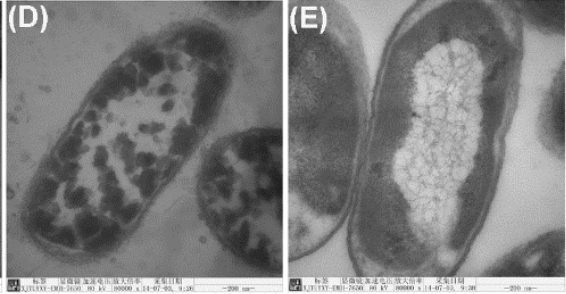

Fig. 5 Transmission electron microscopy images of ESBL-producing K. pneumoniae. (A): without any treatment, (B): incubated with $10 \mathrm{mM} 5$-ALA at $37^{\circ} \mathrm{C}$ for $4 \mathrm{~h}$ in the dark, (C): incubated with $10 \mathrm{mM} \mathrm{MAL}$ at $37^{\circ} \mathrm{C}$ for $4 \mathrm{~h}$ in the dark, (D): incubated with $10 \mathrm{mM}$ 5-ALA and irradiated with 400-780 nm white light for $60 \mathrm{~min}$, and (E): incubated with $10 \mathrm{mM}$ MAL and irradiated with $400-780 \mathrm{~nm}$ white light for $60 \mathrm{~min}$. 

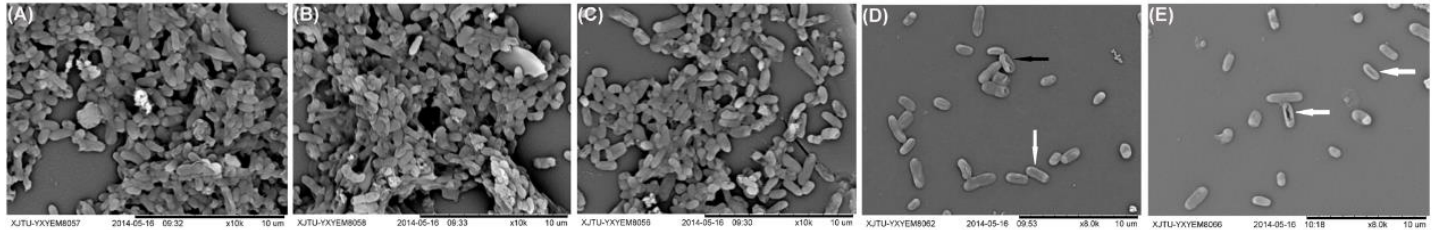

Fig. 6 Scanning electron microscopy images of ESBL-producing K. pneumoniae. (A): without any treatment, (B): incubated with $10 \mathrm{mM} \mathrm{5-ALA} \mathrm{at} 37^{\circ} \mathrm{C}$ for $4 \mathrm{~h}$ in the dark, (C): incubated with $10 \mathrm{mM}$ at $37^{\circ} \mathrm{C}$ for $4 \mathrm{~h}$ MAL in the dark, (D): incubated with 10 $\mathrm{mM}$ 5-ALA and irradiated with 400-780 $\mathrm{mm}$ white light for $60 \mathrm{~min}$, and (E): incubated with $10 \mathrm{mM}$ MAL and irradiated with $400-780 \mathrm{~nm}$ white light for $60 \mathrm{~min}$. 


\title{
Photodynamic Inactivation of Klebsiella pneumoniae Biofilms and Planktonic cells by 5-Aminolevulinic Acid and
}

\section{5-Aminolevulinic Acid Methyl Ester}

\author{
Chengcheng Liu ${ }^{1}$, Yingli Zhou ${ }^{2}$, Li Wang ${ }^{2}$, Lei Han ${ }^{1}$, Jin'e Lei ${ }^{1,2}$, Hafiz \\ Muhammad Ishaq ${ }^{1}$, Jiru Xu ${ }^{1, *}$, Sean P. Nair ${ }^{3, *}$
}

${ }^{1}$ Department of Immunology and Pathogenic Biology, College of Medicine, Xi'an Jiaotong University, 76 West Yanta Road, Xi'an 710061, P. R. China.

2 The First Affiliated Hospital of College of Medicine, Xi' an Jiaotong University, 277 West Yanta Road, Xi'an 710061, P. R. China.

${ }^{3}$ Department of Microbial Diseases, UCL Eastman Dental Institute, 256 Gray's Inn Road, London WC1X 8LD, UK

* Corresponding author: Jiru Xu (E-mail: xujiru@mail.xjtu.edu.cn); Sean P. Nair (E-mail: sean.nair@ucl.ac.uk) 
Dear Prof. Keyvan Nouri and Reviewers:

Thank you very much for your letter and for the reviewer's good comments on our manuscript entitled "Photodynamic Inactivation of Klebsiella pneumoniae and Biofilms by 5-Aminolevulinic Acid and 5-Aminolevulinic Acid Methyl Ester" (LIMS-D-14-00456). These comments are all valuable and have been helpful to us for improving the manuscript. We have addressed all of the comments made by the reviewers and incorporated their suggested. The main corrections in the paper and the responses to the reviewer's comments are as follows:

Responses to the reviewers' comments:

Reviewer \#2:

1. "It seems to me that this paper reports a well done experimental work. The authors have used good approaches to determine the effect of PDT on Klebsiella pneumoniae. Moreover most of the initial criticisms have been answered by the authors. Now my main concern is derived from the fact that Klebsiella pneumoniae is a close microorganism to Escherichia coli. Thus, it should be expected an identical (or highly similar) behavior when treated with an identical PS and light, as the authors found. In other words the description of the effect of 5-ala mediated PDT on the different Enterobacteriaceae species could initiate a long long series of "salami" papers just determining their quantitative effect in each species. Enterobacteriaceae includes more than 30 genera and more than one hundred species and probably their susceptibility to PDT is quite similar. I believe the authors should highlight where is the novelty of this paper, or a stronger rationale. Alternatively they can compare the effect on different species and discuss... but I think this is not the case."

$\mathrm{R}$ : We thank the reviewer for the important comment on the close evolutionary relationship between $K$. pneumoniae and E. coli which might lead one to conclude 
that any study of PDI of K. pneumoniae would be similar to studies on PDI of E. coli. We should have made it clearer in the original manuscript that there is one study looking at 5-ALA mediated PDI of $K$. pneumoniae which showed that it was not effective. In addition the literature shows that there are differences in the susceptibility of different $E$. coli strains to 5-ALA mediated PDI. We have modified the text of the manuscript to emphasis this in the introduction and discussion. Our results on 5-ALA mediated PDI of K. pneumoniae also suggest that this bacterium is less susceptible to PDI than E. coli and we mention this in the discussion as well.

Our study is also novel because the capacity of 5-ALA to mediate PDI of biofilms of these organisms has not been reported. We show that 5-ALA mediated PDI of biofilms depends on the capacity of the bacterium to form a biofilm and that this treatment not only kills the bacteria but also disrupts the biofilm.

\section{Electron microscopy.}

2. "I cannot see injuries in the wall in $(E)$. If the authors do they should include a sentence to describe. It would be better to include pictures with more than one single cell. I've some experience in TEM of bacteria and there is certain heterogeneity; thus, images are suggestive when one can see various individuals or when statistical analysis is done (this is time consuming and actually unnecessary). On the other hand ultrathin sections should be observed taking into account that one van visualize sections in which the genome cannot be seen because it was below the section. In my opinion the unique image reported by authors that gives significant information is (D) because the condensation of cytoplasmic components."

$\mathrm{R}$ : We have rewritten this part of the results section as requested by the reviewer. When we were performing this experiment, most cells seen in different fields were very similar to those shown in image (E). The cellular structure differences in image (D) and (E) have been described in the text of the results section and the discussion. We have also investigated the capacity of 5-ALA and MAL to mediate PDI of 
vancomycin-resistant Enterococci (VRE), and seen similar cell structure changes. We do not know the reason for these differences.

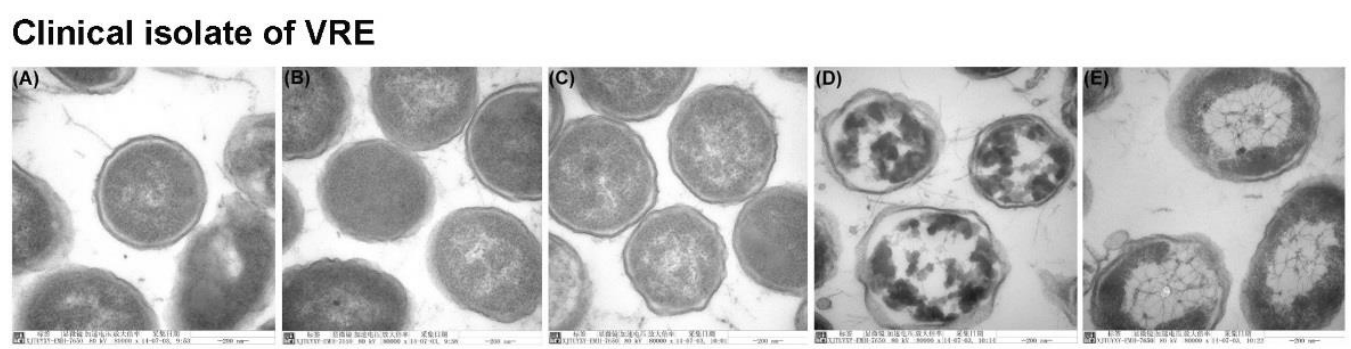

E. faecalis (ATCC 51299)

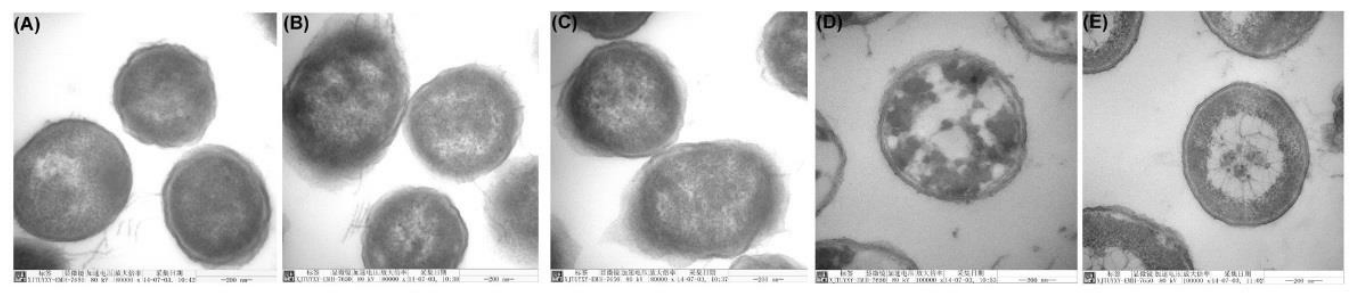

TEM images of the clinical isolate of VRE and E. faecalis (ATCC 51299). (A): without any treatment, (B): incubated with $10 \mathrm{mM} 5$-ALA at $37^{\circ} \mathrm{C}$ for $4 \mathrm{~h}$ in the dark, (C): incubated with $10 \mathrm{mM} \mathrm{MAL}$ at $37{ }^{\circ} \mathrm{C}$ for $4 \mathrm{~h}$ in the dark, (D): treated with 10 mM 5-ALA and irradiated for $60 \mathrm{~min},(\mathrm{E})$ : treated with $10 \mathrm{mM}$ MAL and irradiated for $60 \mathrm{~min}$.

\section{Scanning microscopy}

3. "Images of SEM are also difficult to be interpreted. I assume that initially all biofilms had the same age before treatment. Whereas in $\mathrm{A} B$ and $\mathrm{C}$ one can observe "normal biofilms" in $D$ and $E$ there is not biofilm, but only a few individuals; this means that biofilm has been physically removed. This is much more relevant than one cell/electronmicrograph exhibiting a certain abnormal shape. But I cannot understand by which mechanism biofilm was removed. Authors should add an explanation."

R: We agree SEM images can be difficult to be interpreted. The biofilms are all of the same age. The reviewer's interpretation of the SEMs showing that biofilms have been 
disrupted is correct and that this is an important finding has been emphasized in the revised manuscript.

4. "Despite my native tongue is not English I believe the English style can be improved a little. Some sentences remain unclear."

$\mathrm{R}$ : The manuscript has been revised throughout to improve the English language and style.

\section{Reviewer \#3:}

1. "I like the article, it has interesting useful results for researchers in the field. I would recommend however for future experiments not to perform all experiments in buffer, but in more realistic protein rich environments. Additionally, I am not sure whether UV absorbance is the best way to estimate membrane injuries."

$\mathrm{R}$ : Special thanks to you for the good comments and suggestions. In future work we will perform the PDI experiments in more protein rich environments. However given that in 5-ALA mediated PDI, the active PS is endogenous and located within the cytoplasm of the bacterium such an environment may not affect the outcome very much. The question raised by the reviewer as to the use of UV absorbance being the best method to estimate membrane injury is valid. It would not be the best method to accurately quantify the amount of membrane damage but it is easy to perform and useful for estimating/ establishing that membrane damage is occurring. It is a method that is used in studies such as ours. For example Spesia et al. used UV absorbance at $260 \mathrm{~nm}$ to estimate membrane injuries in the photodynamic inactivation of Escherichia coli and Streptococcus mitis [1,2]. Chen and Je also used the method to detect membrane injuries of bacteria treated with dendrimer biocides and chitin derivative $[3,4]$. 
1. Spesia MB et al (2009) Mechanistic insight of the photodynamic inactivation of Escherichia coli by a tetracationic zinc(II) phthalocyanine derivative. Photodiagn Photodyn Ther 6 (1): 52-61

2. Spesia MB, Duraniti EN (2013) Photodynamic inactivation mechanism of Streptococcus mitis sensitized by zinc (II) 2, 9, 16, 23-tetrakis [2-(N, N, $N$-trimethylamino) ethoxy] phthalocyanine. J Photochem Photobiol B 125 (5): $179-187$

3. Chen CZ, Cooper SL (2002) Interactions between dendrimer biocides and bacterial membranes. Biomaterials 23 (16): 3359-3368

4. Je JY, Kim SK (2006) Antimicrobial action of novel chitin derivative. Biochim Biophys Acta 1760 (1): 104-109

We appreciate for your and the reviewers' work on critically reading our manuscript and offering us constructive feedback which we believe has improved what we have written. We hope that the corrections will meet with approval.

Once again, thank you very much for your comments and suggestions.

Thank you and best regards!

Yours sincerely,

Prof. Jiru Xu

College of Medicine

Xi' an Jiaotong University

76 West Yanta Road

Xi'an 710061, P. R. China. 